\title{
Effect of post-activation potentiation induced by one, two or three half-squats on repeated sprint acceleration performance
}

\author{
Mehdi Rouissi \\ Olfa Turki ${ }^{1,2}$ \\ Nicola Luigi Bragazzi ${ }^{3}$ \\ Adam Owen ${ }^{4,5}$ \\ Monoem Haddad 7 \\ Karim Chamari6 \\ Moktar Chtara1,2
}

1 Tunisian Research Laboratory "Sport Performance Optimisation", National Center of Medicine and Science in Sports, Tunis, Tunisia

2 Department of Sports and Physical Activities, Higher Institute of Sport and Physical Education of Ksar Saïd, Manouba, Tunisia

3 School of Public Health, Department of Health Sciences (DISSAL), University of Genoa, Genoa, Italy

4 BenficaLAB, SL Benfica, Lisbon, Portugal.

5 Centre de Recherche et d'Innovation sur le Sport, Université Claude Bernard Lyon.1, Lyon, France.

6 Athlete Health and Performance Research Centre, ASPETAR, Qatar Orthopaedic and Sports Medicine Hospital, Doha, Qatar

7 Sport Science Program, College of Arts and Sciences, Qatar University, Doha, Qatar

Corresponding author:

Nicola Luigi Bragazzi

School of Public Health, Department of Health

Sciences (DISSAL), University of Genoa,

Genoa, Italy

E-mail: robertobragazzi@gmail.com

\section{Summary}

Introduction: The aim of this study was to analyze the effect of different post-activation potentiation (PAP) protocols on initial-acceleration (0-10 $\mathrm{m})$ and late-acceleration phases $(10$ to $30 \mathrm{~m})$ within a repeated sprint ability (RSA) test.

Methods: Twenty athletes (age: $20.8 \pm 1.2$ years, height: $180.2 \pm 5.3 \mathrm{~cm}$, body mass: $76.8 \pm 6.4 \mathrm{~kg}, \%$ body fat: $10.9 \pm 2.8$, and 3 repetition maximum [3RM] of half-squats $152.9 \pm 14.8 \mathrm{~kg}$ ) completed 4 testing sessions of RSA testing (7x30-m sprints, starting every $25 \mathrm{~s}$, with an active recovery in- between). Five minutes before the RSA-testing, conditioning protocols were performed: I) one halfsquats at $90 \%$ of 1 repetition maximum (1RM) [PAP 1 ; II) two half-squats at $90 \%$ of 1 RM [PAP [P $^{2}$;II) three half-squats at $90 \%$ of $1 \mathrm{RM}\left[\mathrm{PAP}_{3}\right]$, and (IV) the control protocol [CON]: no effort. Each conditioning condition was applied in a counterbalanced, randomized order on separate days separated by a minimum of 72 hours' rest.

Results: ANOVA showed that $\mathrm{PAP}_{1}$ and $\mathrm{PAP}_{2}$ sessions were similar, and brought significantly improved results for: $0-30 \mathrm{~m}$ and $0-10 \mathrm{~m}$ sprints of the RSA-time $\left(p<0.001, E S=\right.$ large) $v s$ the $\mathrm{PAP}_{3}$ and CON-conditions. For the late-acceleration phase of the RSA, the conditioning activity gave no effect ( $p>0.05, E S=s m a l l)$. Furthermore, magnitude-based inference revealed that both $\mathrm{PAP}_{1}$ and $\mathrm{PAP}_{2}$ protocols elicited changes $>75 \%$ likelihood of exceeding the smallest worthwhile change $(>99 \%$ likely) for mean sprint-time (RSA mean) and the percentage of sprint-decrement $\left(R S A_{d e c}\right)$ in overall 0-30 $\mathrm{m}$ and $0-10 \mathrm{~m}$ of the RSA test.

Conclusion: $\mathrm{PAP}_{1}$ and $\mathrm{PAP}_{2}$ exert a positive effect on the initial-acceleration phase of the RSA and could be considered in the preparation routine of repeated sprinting activities.

KEY WORDS: PAP, soccer players, sprint, strength, team-sport.

\section{Introduction}

Field-based team sports generally consist of multiple intermittent bouts of near-maximal or high-speed bouts $^{1,2}$ that are termed as repeated sprint ability $(\mathrm{RSA})^{2}$ and represent a critical component of physical fitness ${ }^{3,4}$. Previous literature reported that maximal or near-maximal muscular actions may acutely improve subsequent neuromuscular performance by inducing a phenomenon known as post-activation potentiation (PAP) ${ }^{5,6}$. PAP refers to the phenomenon by which the acute contractile ability of a muscle is enhanced in response to a conditioning stimulus such as a heavy resistance exercise (HRE).

From a practical standpoint, PAP has been suggested to exploit the enhancement of athletic performance $^{7}$, however, the responses to PAP seem to be highly individualized based on responders vs non-re- 
sponders ${ }^{8,9}$. Literature has suggested that with several players positively responding to PAP protocols, others players may not significantly improve their performance with this method ${ }^{8,9}$. The efficacy by which a conditioning activity can stimulate PAP mechanisms and acutely enhance muscular performance ultimately depends on the balance between PAP and fatigue which may be affected by numerous factors including the rest interval between PAP and the effort required $^{10}$, training experience ${ }^{11}$, intensity ${ }^{5}$ and the volume of PAP ${ }^{12}$.

Many studies reported the positive effect of PAP protocols on linear sprinting performance ${ }^{13-15}$. However, only few studies have investigated the occurrence of PAP on intermittent sprint performance ${ }^{16-18}$. Okuno et al. ${ }^{18}$ concluded that a high intensity squat exercise may be used as acute intervention for improving RSA amongst elite handball players. In a recent study conducted by McLaren et al. ${ }^{17}, 29$ college-aged male field-sport athletes performed four repetitions of back squats $(90 \%$ $1 \mathrm{RM}$; control=20\% $1 \mathrm{RM}$ ), rested $8 \mathrm{~min}$, performed a set of $4 \times 40-m$ sprints with $55 \mathrm{sec}$ inter-repetition active recovery, and rested for $8 \mathrm{~min}$ after the last sprint. This was performed two more times, for a total of three sets (of back squats and sprints) performed 20 min apart. Subjects ran significantly faster after PAP and the PAP effect lasted up to $11 \mathrm{~min}$ after heavy back squats and was repeated successfully three times.

Duncan et al. ${ }^{16}$ reported that a PAP protocol consisting in back squats performed at $90 \% 1 \mathrm{RM}$, administered four min prior to $7 \times 30-\mathrm{m}$ sprint separated by $25 \mathrm{sec}$, could reduce the fatigue index in a sample of ten male professional Rugby Union players.

Although the effect of PAP on overall RSA performance has been investigated, to the best of our knowledge, this is the first study investigating systematically the effect of PAP on the initial and late acceleration phases of a repeated sprint test, potentially enabling to understand when the improvement of the overall RSA performance occurs. This may help coaches in training session designing ad hoc PAP-based training programs. As a result, the main purpose of the present study was to investigate the effects of various PAP. based protocols on the initial $(0-10 \mathrm{~m})$ and late acceleration phases $(10-30 \mathrm{~m})$ of a RSA test. The second aim was to examine the variation in individual responses to PAP protocols.

\section{Materials and methods}

\section{Participants}

This study was conducted on twenty male elite-level field soccer-players, randomly chosen among members of the first division soccer teams of the Tunisian National League 1 (age: $20.8 \pm 1.2$ years, height: $180.2 \pm 5.3 \mathrm{~cm}$, body mass: $76.8 \pm 6.4 \mathrm{~kg}, \%$ body fat: $10.9 \pm 2.8$, and 3 repetition maximum [3RM] halfsquats: $152.96 \pm 14.85 \mathrm{~kg}$ ). All the participants had routinely performed half-squats (i.e. 90-degree angle in the knee joint between femur and tibia) and deadlifts as part of their regular resistance training regime for a minimum of 2 years before the study. The players were all starters in the competitive season (fourth month of the season) when the testing was conducted. They participated in a regular training and soccer competition schedule for at least 8 years before the experiment. They trained 5-6 times a week ( 90 minutes per session) with a competitive match taking place during the weekend in their national-level championships. All the participants gave their informed consent before the investigation. The study was conducted according to the declaration of Helsinki, and the protocol was fully approved by the institutional Ethics Committee of the National Centre of Medicine and Science of Sports of Tunis (CNMSS). During all the experimental sessions, the athletes were given standardized instructions and verbal encouragements to perform to the best of their ability. Furthermore, the guidelines of the journal were taken into account ${ }^{19}$.

\section{Procedures}

One week before the commencement of the study, all players attended 2 orientation sessions. The first day was dedicated to anthropometric measurements (age, height, body mass, body fat \%) and determine the participants' $3 \mathrm{RM}^{20}$. The second day was used for familiarization to the RSA test. After these 2 condition sessions, the participants returned to the university's resistance training room on 4 separate occasions for the testing intervention sessions, each separated by 72 hours. Sessions were administered randomly and in a counter-balanced order and involved the participant performing either the three potentiation protocols $\left(\mathrm{PAP}_{1}, \mathrm{PAP}_{2}\right.$, and $\left.\mathrm{PAP}_{3}\right)$ or the control (C) test intervention.

Each session began with the subjects performing a standardized general warm-up for 15 min including light intensity jogging, series of dynamic stretching and several acceleration runs. The RSA-test was performed on a $3^{\text {rd }}$ generation synthetic soccer turf at the same time of day (time: $\sim 15: 00 \mathrm{~h}$, temperature: $\sim 21.6 \pm 2.1^{\circ} \mathrm{C}$, relative humidity: $\sim 69.9 \pm 0.2 \%$, with no wind and no rain). All players were asked to wear adapted soccer boots, which allow them to have good adherence to the pitch. Figure 1 illustrates the study design.

\section{Testing sessions}

\section{Preliminary testing}

The preliminary testing session was used to determine the participant's height, body mass, and percentage body fat. Height and body mass were measured using a stadiometer to the nearest $0.5 \mathrm{~cm}$ and a standard electronic scale (accurate to $0.01 \mathrm{~kg}$ ), respectively. Skinfold thickness was measured to the nearest 0.2 $\mathrm{mm}$ at four predetermined sites (biceps, triceps, subscapular, and suprailiac) using Harpenden skinfold callipers (Lange, Cambridge, MA, USA). To increase measurement reliability, the skinfold sites were measured 3 times by the same investigator, with the 


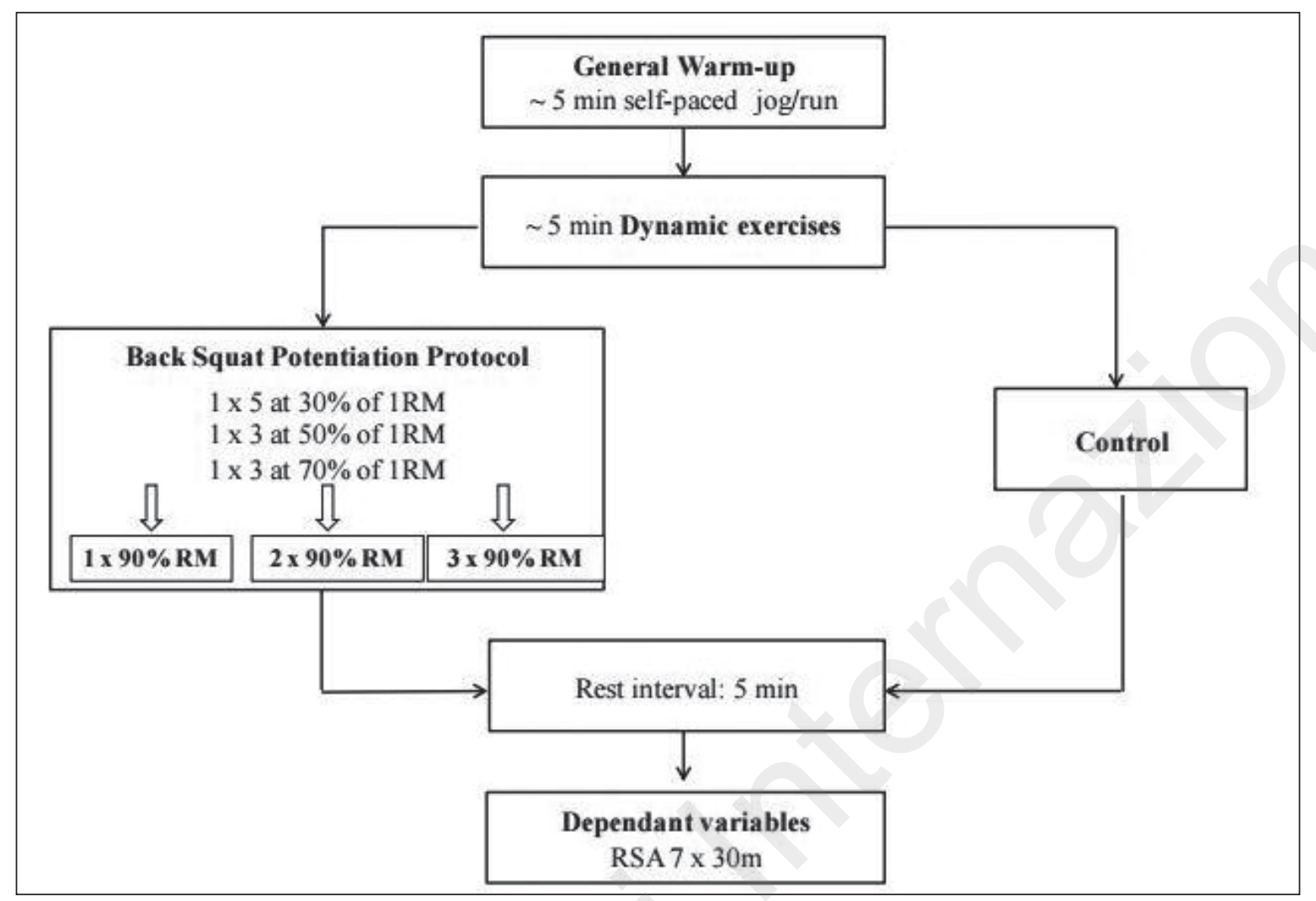

Figure 1. Summary of research design.

average value used for data analysis. Percentage of body fat was estimated using the equations described by Durnin and Womersley (1974). The participants' 3RM was tested using the procedure outlined by the National Strength and Conditioning Association ${ }^{20}$. Based on this first testing day, the value of 1RM was estimated for each participant, according to the percentage 1RM-repetition relationship outlined by ${ }^{20}$. Before the start of the strength-testing session, all participants underwent a standardized general warmup that comprised light intensity jogging for $5 \mathrm{~min}$, followed by a weights specific warm-up involving 8 repetitions at $50 \% 1 \mathrm{RM}, 4$ repetitions at $70 \% 1 \mathrm{RM}$, and finally 2 repetitions at $80 \%$ of $1 \mathrm{RM}$, with 3 -min intervals between them. Each participant used their estimated $1 \mathrm{RM}$ as a guide. After the final warm-up set, participants attempted 3 repetitions of a set load (3RM), and if successful, the lifting weight was increased until the participant could not lift the weight through the full range of motion. A 5-min rest was imposed between all attempts. The 3RM was determined after 3-4 attempts in all participants. The reliability of the $3 \mathrm{RM}$ half-squats was tested over a 1week period before experimentation (ICC=0.96).

\section{Protocol for eliciting PAP}

All participants underwent a standardized general warm-up for 15 min including light intensity jogging, series of dynamic stretching and several acceleration runs $^{21-23}$. Before PAP protocols, players performed specific warm-up involving eight half-squats at $50 \%$ of $1 \mathrm{RM}$, four half-squats at $70 \%$ of $1 \mathrm{RM}$, and finally two half-squats at $80 \%$ of $1 \mathrm{RM}$, with 2 -min intervals between them. The protocol for eliciting consisted of one, two or three half-squats at $90 \%$ of $1 \mathrm{RM}$.

\section{RSA testing}

The participants rested for 5 minutes before performing the RSA test. A 5-min rest was selected on the basis of previously published studies ${ }^{13,23}$ that have reported significant potentiation effects with this rest interval. The RSA test consisted of $7 \times 30$ m maximal straightline sprints with 25-s active recovery on an outdoor synthetic court. During the active recovery, participants slowly jogged back $(\sim 20 \mathrm{~m})$ to the starting line and waited for the next sprint. The starting position (i.e. standing position with the preferred foot forward and placed exactly $0.5 \mathrm{~m}$ behind the starting line) was controlled and consistent over the seven sprints of the RSA test. Sprint time for $30 \mathrm{~m}$ (with 10-m split times) was measured by an infra-red timing system (Brower Timing Systems, Salt Lake City, UT; accuracy of 0.01 seconds) located at the starting and finishing line, and the recovery time was controlled by a simple hand-held stopwatch. The participants stood $0.5 \mathrm{~m}$ behind the sensor before they commenced each sprint, starting from a standing position. Participants were given strong verbal encouragement throughout all trials to ensure 
maximal effort. The timing gate at $10 \mathrm{~m}$ recorded the time for the initial-acceleration phase of the sprint (0$10 \mathrm{~m}$ ). The timing gate at $30 \mathrm{~m}$ stopped the timer and recorded the total time taken to sprint $30 \mathrm{~m}$. Time spent in the late-acceleration phase was calculated by subtracting the initial-acceleration time from the overall time ${ }^{24}$. The following variables were derived from the RSA test: a. best sprint time (the fastest time for 0-10 $\mathrm{m}, 10-30 \mathrm{~m}$, and 0-30 m); b. mean sprint time (the means of all 7 sprints for $0-10 \mathrm{~m}, 10-30 \mathrm{~m}$, and $0-30$ $\mathrm{m}$ ); c. percentage of performance decrement (a measure of the performance decline demonstrated over the entire RSA test for 0-10 m, 10-30 m, and 0-30 $m)^{25}$. The RSA $A_{\text {best }}$, the RSA mean and the RSA dec were determined according to Rampinini ${ }^{3}$ (compared to Bishop).

\section{Statistical analyses}

Means \pm standard deviations (SD) were used to describe variables. Before using parametric tests, the assumption of normality was verified using Kolmogorov-Smirnov test. Reliability of the measures (dependent variables) was assessed with a Cronbach's model intraclass correlation coefficient (ICC) and standard error of measurements (SEM) according to the method of Hopkins ${ }^{26}$. In addition, the effects of post-activation potentiation on RSA performance were evaluated with a 1-way analysis of variance (ANOVA) for repeated measures to compare the 4 conditions. When significant $F$ values were obtained $(p<0.05)$, paired comparisons were used in conjunction with the Holm's Bonferroni method for controlling type I error ${ }^{27}$ to determine significant differences. The effect size was calculated for all ANOVAs with the use of a partial eta squared $\left(n^{2}\right)$ $<0.01=$ small, $0.01-0.06=$ medium, and $>0.06=$ large). In addition to the comparison analyses, Cohen's $d$, smallest worthwhile change (SWC), and likelihood of clinical meaningfulness were calculated for RSA $\mathrm{Aest}_{\text {be }}$ $\mathrm{RSA}_{\text {mean, }}$ and $\mathrm{RSA}_{\mathrm{dec}}{ }^{28}$. The Cohen's $\mathrm{d}$ is calculated from the mean change divided by the SD of the data; thresholds for qualitative descriptors of Cohen's $d$ were set at, $<0.20$ is "trivial," $0.20-0.50$ is "small," $>0.50-0.80$ is "moderate," and $>0.80$ is "large" 29 . The smallest change to be considered worthwhile (SWC) was thus calculated from 0.20 of the SD of the data. The threshold of a clinical meaningful effect was set at $75 \%{ }^{28}$. The quantitative chances of beneficial effects were assessed qualitatively as follows: $<1 \%$ : almost certainly not; $1-5 \%$ : very unlikely, $>5-25 \%$ : unlikely; $>25-75 \%$ : possible; $>75-95 \%$ : likely; >95-99: very likely, and $>99 \%$ almost certain. Descriptive statistics, $p-$ values, 95\% confidence limits, and Cohen's $d$ for the within-participant contrasts were calculated by customwritten Excel spreadsheets (Microsoft Office, 2007). Statistical analyses were performed using SPSS software statistical package (SPSS Inc., Chicago, IL, version. 16.0), and statistical significance was set at $p$ $<0.05$.

\section{Results}

\section{Reliability}

ICCs and SEMs for the mean scores of overall sprint, acceleration, and maximal velocity phases of RSA are presented in Table I. The ICC values for best time and mean time showed "high reliability" (ICC range: 0.980.99; SEM range: 0.02-0.03s).

\section{Analysis with repeated measures}

Mean and standard deviations of RSA mean, $R S A_{\text {best }}$ and $R S A_{d e c}$ of each conditions protocol on each of the dependent variables of overall RSA-time were presented in Table II. In overall 0-30 m RSA-time and initial acceleration phase of the RSA, ANOVA revealed a significant difference between the 4 conditions for RSAmean and RSAdec. The Bonferroni test indicated that the $\mathrm{PAP}_{1}$ and $\mathrm{PAP}_{2}$ sessions' effects were similar, and showed significant positive effects $\left(p<0.001 ; \eta^{2}\right.$ range: 0.42 to 0.62 , large), while the $\mathrm{PAP}_{3}$ and control group did not show any effect. On the contrary, in the lateacceleration phase $(10$ to $30 \mathrm{~m})$ of the RSA there were no significant differences between conditions $(p>0.05)$ for $\mathrm{RSA}_{\text {mean }}, \mathrm{RSA}_{\text {best, }}$ and RSA dec.

\section{Analysis of peak data}

For overall 0-30 m RSA-time, the 2 PAP conditions that elicited a substantial likelihood of potentiating $R S A_{\text {mean }}$ and $R_{S A}$ dec a substantial amount (i.e., had a $>75 \%$ of exceeding a small Cohen's $d$ ) were the $\mathrm{PAP}_{1}$ and $\mathrm{PAP}_{2}$ conditions. For first $10 \mathrm{~m}$ of the RSA, both the $1 \times 90 \%$ $\mathrm{RM}$ and $2 \times 90 \% \mathrm{RM}$ protocol elicited changes that exceed $75 \%$ likelihood of exceeding the SWC (>99\%). For the last $20 \mathrm{~m}$ of the RSA sprints, the RSA mean, $R S A_{\text {best }}$, and $R S A_{\text {dec }}$ were unaffected by any of the stimulus protocols with no protocol eliciting a $75 \%$ likelihood of exceeding the SWC from the control condition (Tab. III).

\section{Individual responses}

Figure 2 shows the results as a percentage of the control protocol performance, with each control performance considered to be $100 \%$ of the individual's maximal performance (i.e., a sprint time of less than $100 \%$ represent an improved performance). Figure 2 illustrates the individual changes of each participant for each RSA parameter and PAP protocol (where no bar appears for a participant, this represents a $0 \%$ change). The graph illustrates that the range of responses by each individual varies between participants, test, and PAP method used. There is a great consistency between the results for the overall RSA and the first $10 \mathrm{~m}$ of the RSA, with patterns emerging on the responders and nonresponders to the PAP protocols. For overall RSA-time, participant numbers 1,8 and 12 had large positive responses after all PAP protocols, especially the $\mathrm{PAP}_{1}$ and $\mathrm{PAP}_{2}$ protocols, with improvements up to $8.6 \%$. However, there were also participants who responded 
Table I. Test-retest reliability of tests.

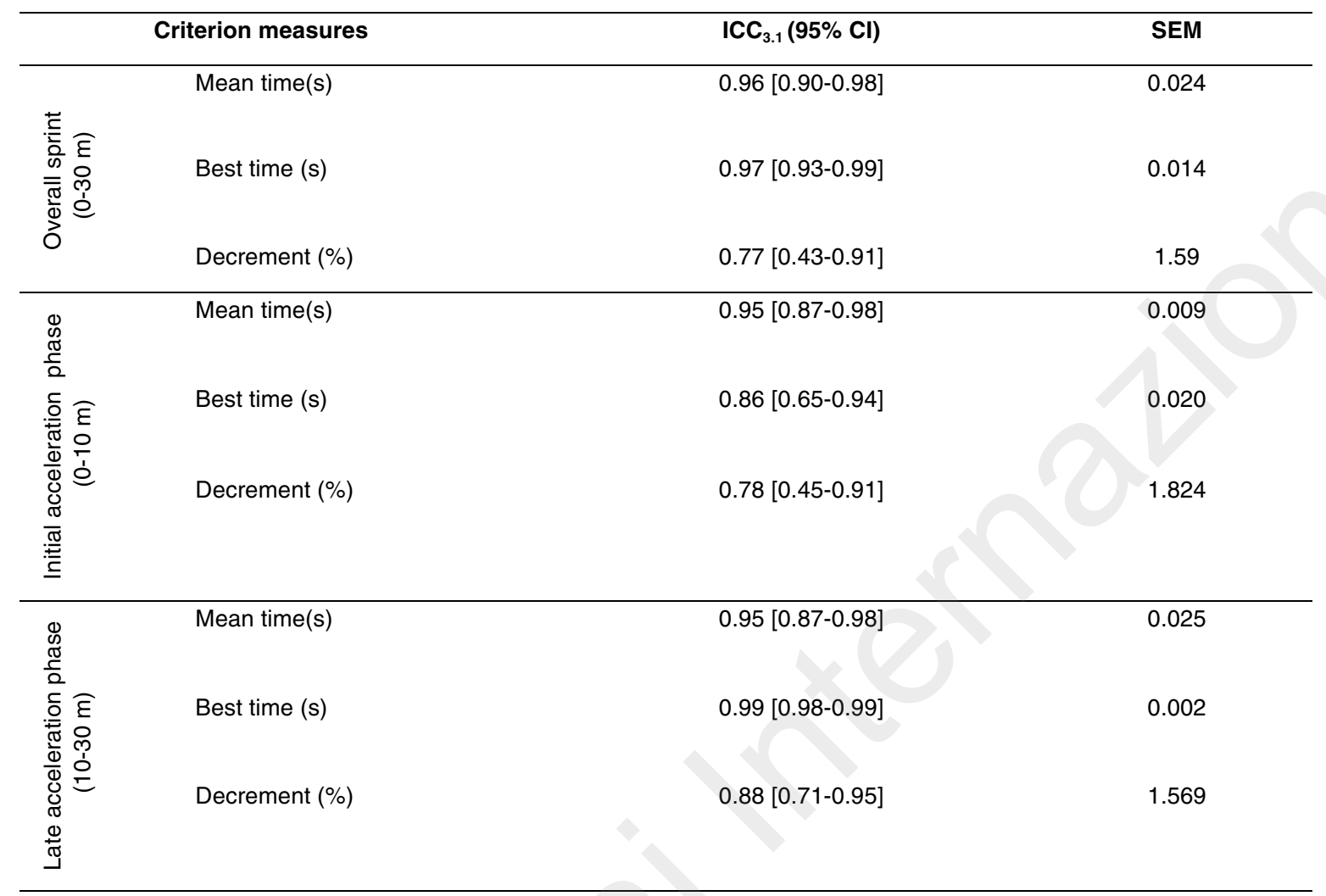

negatively to the PAP protocols. 4.2 to $5.1 \%$ decrease in overall RSA-performance was found for participants' 6,9 , and 20 after the $\mathrm{PAP}_{3}$ protocol. Large individual responses were also evident for $\mathrm{RSA}_{\text {dec. }}$. For the first $10 \mathrm{~m}$ of the RSA-time, participants' 12 and 16 responded largely positively to all PAP protocols ( 7.1 to $10.9 \%)$. Participants' 6 and 9 responded largely negative after $\mathrm{PAP}_{3}$ protocol, with decreases up to $11.8 \%$. In addition, large individual responses were also evident for $\mathrm{RSA}_{\text {dec. }}$.

\section{Discussion}

The purpose of this study was to analyze the effect of different volumes of PAP exercises on the initial (0-10 m) and late (10 to $30 \mathrm{~m}$ ) acceleration phases of a RSA test in elite soccer players. The originality of the present study was the assessment of the effect of PAP protocols during the two acceleration phases of an RSA test. This kind of assessment allows understanding if the potential improvement of the overall RSA performance occurring during the initial and/or the late acceleration phases of the sprints. This result could help coaches when designing PAP-exercises for training.

The most important findings of this study were that $\mathrm{PAP}_{1}(190 \% 1-\mathrm{RM})$ and $\mathrm{PAP}_{2}(2 \times 90 \% 1-\mathrm{RM})$ protocols were similarly effective in improving overall $(0-30 \mathrm{~m})$ and initial-acceleration phase $(0-10 \mathrm{~m})$ of the RSA-test sprints, by bringing statistically significant effect $(p<0.001, E S=$ large $)$ with respect to the $\mathrm{PAP}_{3}(3 \times 90 \%$ 1-RM) and CON-groups in which did not impact performance. On the contrary, in the late-acceleration phase of the RSA, all RSA parameters (RSA best, $R S A_{\text {mean }}$ and $R S A_{d e c}$ ) did not change after the conditioning activity $(p>0.05, E S=s m a l l)$. It has been reported that PAP exercises can induce a significant improvement of high intensity efforts in many sports ${ }^{13,16,18}$. Results of the present investigation were in accordance with scientific literature ${ }^{16,18}$. Indeed, Okuno and Tricoli ${ }^{18}$ explored the changes in RSA performance after heavy load exercise in elite handball players and found a significant improvement (Cohen's $\mathrm{d}$ : small to moderate) in the best sprint time (RSA $\left.A_{\text {best }}\right)$ and mean sprint time (RSA mean). In the same context, Duncan et al. ${ }^{16}$ reported that a heavy resistance exercise stimulus (90\% 1RM) administered four minutes prior to repeated sprints $(7 \times 30-m$ sprints separated by $25 \mathrm{sec}$ ) test can decline the fatigue index seen during subsequent maximal sprinting over 10 and $30 \mathrm{~m}$ in Rugby Union players. According to the extant scientific literature, two main mechanisms are involved in this process: 1) the phosphorylation of myosin regulatory light chains and 2 ) the increase in the recruitment of higher order motor units ${ }^{11,30}$. In that regard, the improvement in RSA performance postPAP in the present study may be attributed to these aforementioned mechanisms. 
Table II. Mean \pm SD values of $R S A_{\text {best }}, R_{\text {SA }}$ and $R S A_{\text {dec }}$ of each conditions protocol on each of the dependent variables $(n=20)$.

\begin{tabular}{|c|c|c|c|c|c|c|c|}
\hline & & \multicolumn{4}{|c|}{ Conditions } & \multirow{2}{*}{$\begin{array}{c}\text { "F" } \\
\text { p-value }\end{array}$} & \multirow{2}{*}{$\begin{array}{c}\text { Effect } \\
\text { Size } \\
\eta^{2}\end{array}$} \\
\hline & & 1x90\% RM & $2 \times 90 \%$ RM & $3 \times 90 \%$ RM & CON & & \\
\hline \multirow{3}{*}{ 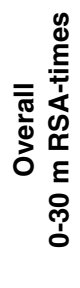 } & Mean time(s) & $4.66 \pm 0.26 ¥$ & $4.64 \pm 0.26 ¥$ & $4.79 \pm 0.25$ & $4.79 \pm 0.28$ & 0.000 & 0.522 \\
\hline & Best time (s) & $4.36 \pm 0.24$ & $4.33 \pm 0.26$ & $4.39 \pm 0.24$ & $4.38 \pm 0.27$ & 0.193 & 0.079 \\
\hline & Decrement (\%) & $6.77 \pm 2.23 ¥$ & $7.09 \pm 2.72 ¥$ & $9.08 \pm 3.34$ & $9.47 \pm 3.62$ & 0.000 & 0.423 \\
\hline \multirow{3}{*}{ 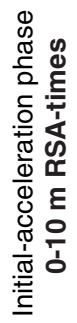 } & Mean time(s) & $1.85 \pm 0.08 ¥$ & $1.84 \pm 0.08 ¥$ & $1.97 \pm 0.08$ & $1.97 \pm 0.09$ & 0.000 & 0.691 \\
\hline & Best time (s) & $1.76 \pm 0.07$ & $1.76 \pm 0.07$ & $1.78 \pm 0.06$ & $1.77 \pm 0.07$ & 0.061 & 0.120 \\
\hline & Decrement (\%) & $5.42 \pm 3.16 ¥$ & $4.92 \pm 2.95 ¥$ & $10.48 \pm 4.37$ & $11.24 \pm 4.46$ & 0.000 & 0.599 \\
\hline \multirow{3}{*}{ 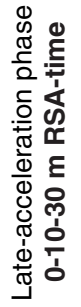 } & Mean time(s) & $2.81 \pm 0.20$ & $2.80 \pm 0.20$ & $2.82 \pm 0.20$ & $2.82 \pm 0.21$ & 0.672 & 0.034 \\
\hline & Best time (s) & $2.58 \pm 0.21$ & $2.58 \pm 0.21$ & $2.57 \pm 0.18$ & $2.58 \pm 0.21$ & 0.897 & 0.010 \\
\hline & Decrement (\%) & $9.00 \pm 4.13$ & $8.78 \pm 3.73$ & $9.97 \pm 4.42$ & $9.44 \pm 4.88$ & 0.263 & 0.067 \\
\hline
\end{tabular}

Significantly different from control, $¥$ Significantly different from $3 \times 90 \% 1 \mathrm{RM}$.

It has been indicated that the relationship PAP-fatigue is influenced by several factors such as: the volume, the intensity, the type of contraction and the type of activity during the PAP ${ }^{11,31-33}$. In the present study, the type and intensity of PAP exercise (i.e. half-squat) and the rest interval separating the PAP from the task, were rigorously controlled. However, the number of halfsquat repetitions (volume) was different between the three PAP protocols. Therefore, the differences in term of volume in the present study may explain the difference of post-PAP performances between protocols. In that regard it has been reported that a moderate volume of stimuli induces more PAP than fatigue when compared to a high volume of stimuli which is linked to the appearance of deleterious fatigue ${ }^{30,34}$. Indeed, it has been reported that a high volume of exercise may induce a higher depletion of phosphocreatine stores when compared to a lower volume of exercise ${ }^{15}$. In conclusion, it is plausible that the high volume of half-squat exercise may explain the aforementioned results. Furthermore, it may be speculated that half-squat exercises superior to three repetitions may negatively affect subsequent RSA performance, and this should be investigated in the future.

The present study showed a between-players variation in term of responses to PAP protocols. The results of the present study were in agreement with the scientific literature. Indeed, it has been indicated that PAP responses are highly individualized (some athletes are responders to PAP however, others athletes are not). In that regard, many studies reported a great variability in PAP responses between athletes suggesting the individualization of PAP protocols in order to obtain benefits among all athletes $33,35,36$. It has been indicated that several factors may contribute to the betweensubject PAP variation. Indeed, in addition to the influence of players' trainability, subjects with a great percentage of fast twitches may be more suitable for PAP benefits ${ }^{10}$. In addition it has been reported that players with a great fatigue resistance (i.e., greater buffering, higher phosphocreatine stores, more oxidative 


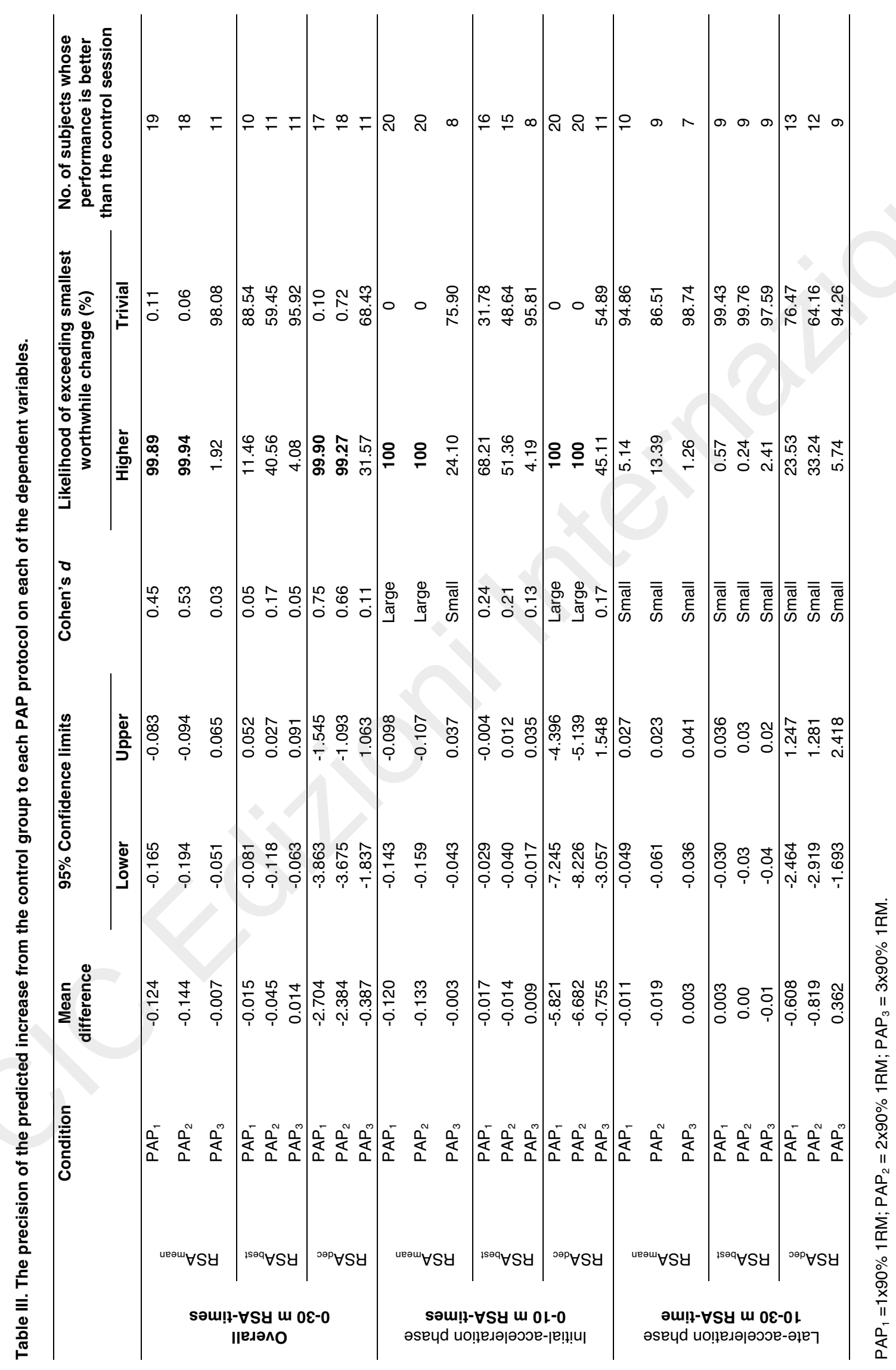




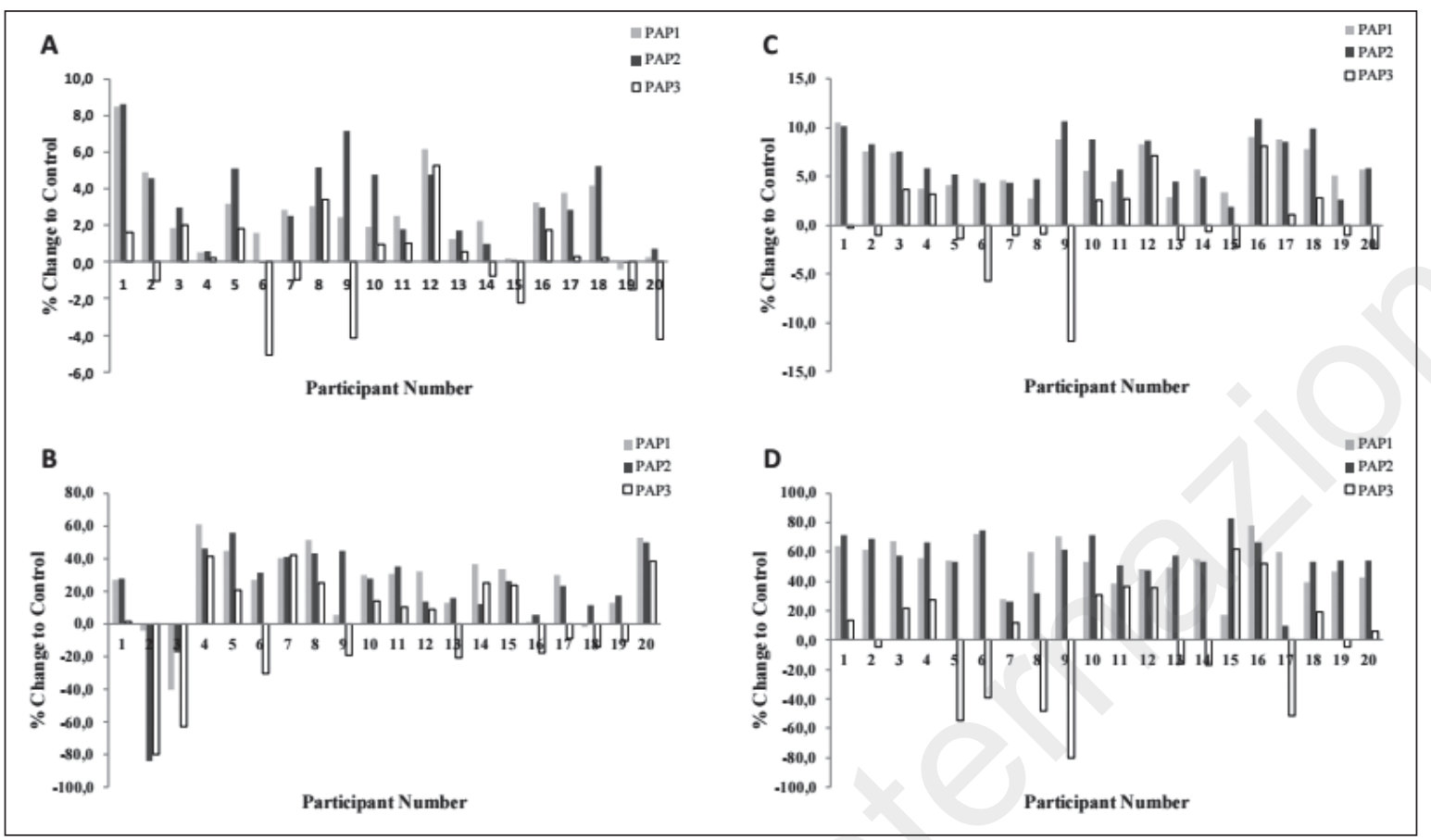

Figure 2. Individual performance changes compared to the control for each PAP protocol for the (A) Overall RSAmean, $(B)$ Overall RSA $A_{d e c},(C)$ Initial-acceleration (0-10 m) phase of the RSA mean and (D) Initial-acceleration (0-10 m) phase of the RSA dec.

enzymes and mitochondria) may allow potentiation to dominate fatigue when compared to players with a low fatigue resistance ${ }^{10,32}$. Furthermore, the positional role of players may also influence the post-PAP responses since many physical and physiological differences are reported between players of different position in soccer ${ }^{36-38}$. In conclusion, the between subject variation following PAP protocols in the present study may be attributed to several factors such as the ones listed here above. However, the design of the present study does not allow confirming such conclusion and therefore future studies in that regard are warranted.

Post-PAP lower limb fatigue was not assessed in the present study which may represent a limit. Such analysis could be useful to determine the level of fatigue generated by each PAP protocol and therefore choosing the appropriate PAP protocol for each player. Therefore, it may be suggested to use a fatigue questionnaire (RPE, for instance), EMG analysis and/or maximal isometric voluntary contraction tests to fix this issue in coming studies.

\section{Conclusion}

The present study revealed that in soccer players, the use of a half-squat warm-up comprising $90 \% 1 \mathrm{RM}$ with 1 or 2 repetitions elicits the appropriate response necessary to augment performance in overall $(0-30 \mathrm{~m})$ RSA-effort compared to three repetitions and no halfsquat. The improvement in RSA was due to an improvement of the initial-acceleration phase $(0-10 \mathrm{~m})$ of the $30 \mathrm{~m}$ repeated sprints.

\section{Practical applications}

Because the potentiation of a prior conditioning program is very individualized, the volume of PAP warm-up activities should be developed individually for each athlete. However, when individual warm-ups are not possible as with a team setting, then a half-squat at $90 \% 1 \mathrm{RM}$ with 1, or 2 repetitions is a time efficient and effective way of maximizing potential response.

\section{Conflict of interest}

There are no conflicts of interest concerning this paper.

\section{Acknowledgements}

This study Authors have no conflict of interest with the procedures used in this research design. No financial support was provided for the completion of any part of this study.

\section{References}

1. Safdar A, Papadopoulos EB, Young JW. Breakthrough Scedosporium apiospermum (Pseudallescheria boydii) brain abscess during therapy for invasive pulmonary aspergillosis following high-risk allogeneic hematopoietic stem cell transplantation. Scedosporiasis and recent advances in antifungal therapy. Transpl Infect Dis. 2002;44:212-217.

2. Spencer M, Bishop D, Dawson B, Goodman C. Physiological and metabolic responses of repeated-sprint activities: specific 
to field-based team sports. Sports Med. 2005;3512:10251044.

3. Rampinini E, Bishop D, Marcora SM, Ferrari Bravo D, Sassi R, Impellizzeri FM. Validity of simple field tests as indicators of match-related physical performance in top-level professional soccer players. Int J Sports Med. 2007;283:228-235.

4. Schimpchen J, Skorski S, Nopp S, Meyer T. Are "classical" tests of repeated-sprint ability in football externally valid? A new approach to determine in-game sprinting behaviour in elite football players. J Sports Sci. 2016;346:519-526.

5. Sale DG. Postactivation potentiation: role in human performance. Exerc Sport Sci Rev. 2002;303:138-143.

6. Dello lacono A, Padulo J, Eliakim A, Gottlieb R, Bareli R, Meckel Y. Post-activation potentiation effects on vertical and horizontal explosive performances of young handball and basketball athletes. J Sports Med Phys Fitness. 2016;56:1455-1464.

7. Seitz LB, Haff GG. Factors Modulating Post-Activation Potentiation of Jump, Sprint, Throw, and Upper-Body Ballistic Performances: A Systematic Review with Meta-Analysis. Sports Med. 2016;462:231-240.

8. Chaouachi A, Poulos N, Abed F, Turki O, Brughelli M, Chamari $\mathrm{K}$, et al. Volume, intensity, and timing of muscle power potentiation are variable. Appl Physiol Nutr Metab. 2011;365:736747.

9. Ruben RM, Molinari MA, Bibbee CA, Childress MA, Harman MS, Reed KP, et al. The acute effects of an ascending squat protocol on performance during horizontal plyometric jumps. J Strength Cond Res. 2010;242:358-369.

10. Tillin NA, Bishop D. Factors modulating post-activation potentiation and its effect on performance of subsequent explosive activities. Sports Med. 2009;392:147-166.

11. Kilduff LP, Bevan HR, Kingsley MI, Owen NJ, Bennett MA, Bunce $\mathrm{PJ}$, et al. Postactivation potentiation in professional rugby players: optimal recovery. J Strength Cond Res. 2007;214 1134-1138.

12. Chiu LZ, Fry AC, Weiss LW, Schilling BK, Brown LE, Smith SL. Postactivation potentiation response in athletic and recreationally trained individuals. J Strength Cond Res. 2003;174:671677.

13. Chatzopoulos DE, Michailidis CJ, Giannakos AK, Alexiou KC, Patikas DA, Antonopoulos CB, et al. Postactivation potentiation effects after heavy resistance exercise on running speed. J Strength Cond Res. 2007;214:1278-1281.

14. McBride JM, Nimphius S, Erickson TM. The acute effects of heavy-load squats and loaded countermovement jumps on sprint performance. J Strength Cond Res. 2005;194:893-897.

15. Turner AP, Bellhouse S, Kilduff LP, Russell M. Postactivation potentiation of sprint acceleration performance using plyometric exercise. J Strength Cond Res. 2015;292:343-350.

16. Duncan MJ, Thurgood G, Oxford SW. Effect of heavy back squats on repeated sprint performance in trained men. $J$ Sports Med Phys Fitness. 2014;542:238-243.

17. McLaren T, King DL, Sforzo G. Sustainability and repeatability of post-activation potentiation. J Sports Med Phys Fitness. 2016.

18. Okuno NM, Tricoli V, Silva SB, Bertuzzi R, Moreira A, Kiss MA Postactivation potentiation on repeated-sprint ability in elite handball players. J Strength Cond Res. 2013;273:662-668.

19. Padulo J, Oliva F, Frizziero A, Maffulli N. Muscles, Ligaments and Tendons Journal - Basic principles and recommendations in clinical and field science research: 2016 update. MLTJ. 2016;6(1):1-5.

20. Baechle TR, Earle RW, National Strength and Conditioning Association. Essentials of strength training and conditioning. 3. ed. Champaign, IL: Human Kinetics. 2008. XIV, 641 s. p.

21. Tan D, Dawson B, Peeling P. Hemolytic effects of a footballspecific training session in elite female players. Int J Sports Physiol Perform. 2012;73:271-276.

22. Turki O, Chaouachi A, Drinkwater EJ, Chtara M, Chamari K, Amri M, et al. Ten minutes of dynamic stretching is sufficient to potentiate vertical jump performance characteristics. J Strength Cond Res. 2011;259:2453-2463.

23. Stone MH, Sands WA, Pierce KC, Ramsey MW, Haff GG. Power and power potentiation among strength-power athletes: preliminary study. Int J Sports Physiol Perform. 2008;31:55-67.

24. Nikolaidis PT, Dellal A, Torres-Luque G, Ingebrigtsen J. Determinants of acceleration and maximum speed phase of repeated sprint ability in soccer players: a cross-sectional study. Science \& Sports. 2015;301:7-16.

25. Fitzsimons M, Dawson B, Ward D, Wilkinson A. Cycling and running tests of repeated sprint ability. Aust J Sci Med Sport. 1993:2582-2587.

26. Hopkins WG. Measures of reliability in sports medicine and science. Sports Med. 2000;301:1-15.

27. Holm S. A simple sequentially rejective multiple test procedure. Scandinavian Journal of Statistics. 1979;6:65-70.

28. Liow DK, Hopkins WG. Velocity specificity of weight training for kayak sprint performance. Med Sci Sports Exerc. 2003;357: 1232-1237.

29. Cohen J. Statistical power analysis for the behavioural sciences. L Erbraum Associates, Hillside, NJ. 1988:23-97.

30. Hamada T, Sale DG, MacDougall JD, Tarnopolsky MA Postactivation potentiation, fiber type, and twitch contraction time in human knee extensor muscles. J Appl Physiol (1985). 2000;886:2131-2137.

31. Babault N, Maffiuletti NA, Pousson M. Postactivation potentiation in human knee extensors during dynamic passive movements. Med Sci Sports Exerc. 2008;404:735-743.

32. Behm DG, Button DC, Barbour G, Butt JC, Young WB. Conflicting effects of fatigue and potentiation on voluntary force. $J$ Strength Cond Res. 2004;182:365-372.

33. Till KA, Cooke $C$. The effects of postactivation potentiation on sprint and jump performance of male academy soccer players. J Strength Cond Res. 2009;237:1960-1967.

34. Hamada T, Sale DG, MacDougall JD, Tarnopolsky MA. Interaction of fibre type, potentiation and fatigue in human knee extensor muscles. Acta Physiol Scand. 2003;1782:165-173.

35. Evetovich TK, Conley DS, McCawley PF. Postactivation potentiation enhances upper- and lower-body athletic performance in collegiate male and female athletes. J Strength Cond Res. 2015;292:336-342.

36. Lim JJ, Kong PW. Effects of isometric and dynamic postactivation potentiation protocols on maximal sprint performance. $J$ Strength Cond Res. 2013;2710:2730-2736.

37. Bloomfield J, Polman R, O'Donoghue P. Physical demands of different positions in FA Premier League soccer. J Sports Sci Med. 2007;61:63-70.

38. Stolen T, Chamari K, Castagna C, Wisloff U. Physiology of soccer: an update. Sports Med. 2005;356:501-536. 